PROCEEDINGS OF THE

AMERICAN MATHEMATICAL SOCIETY

Volume 136, Number 12, December 2008, Pages 4405-4412

S 0002-9939(08)09362-3

Article electronically published on August 4, 2008

\title{
EF-EQUIVALENT NOT ISOMORPHIC PAIR OF MODELS
}

\author{
SAHARON SHELAH
}

(Communicated by Julia Knight)

\begin{abstract}
We construct non-isomorphic models $M, N$, e.g. of cardinality $\aleph_{1}$, such that in the Ehrenfeucht-Fraissé game of any length $\zeta<\omega_{1}$ the isomorphism player wins.
\end{abstract}

\section{INTRODUCTION}

About 20 years ago, Heikki Tuuri in his thesis Tur90 supervised by Väänänen, asked the following question (for length $<\omega^{3}$ consistently the answer is yes).

0.1. Question. Are there models $M, N$ that are EF-equivalent for the game of length

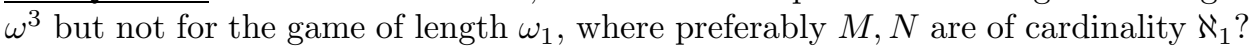

On the history see Väänänen Va95, which asked the question. Subsequently Sh 836. showed that for most regular $\lambda$ we have:

$(*)_{\lambda} \quad$ there are models $M, N$ of cardinality $\lambda$ such that

(a) for any ordinal $\zeta<\lambda$ in the Ehrenfeucht-Fraissé game of length $\zeta$ for the pair $(M, N)$, the isomorphism player wins;

(b) $M, N$ are not isomorphic.

By "most regular $\lambda$ " we mean $\lambda=\lambda^{\aleph_{0}}$. This was continued in Havlin and Shelah HvSh 866], which proved it for "almost" all regular $\lambda$ : if $\lambda \geq \beth_{\omega}$ or if $\lambda>2^{\aleph_{0}}$, assuming a very weak statement in pcf theory, quite possibly provable in ZFC. However, if $\lambda=\aleph_{1}<2^{\aleph_{0}}$, this does not help, so the problem as stated remained open.

Here at last the question as stated is given a positive answer.

We construct a pair of non-isomorphic models of cardinality $\aleph_{1}$ which are equivalent for the EF-game of length $\zeta$ iff $\zeta<\omega_{1}$. We then prove $(*)_{\lambda}$ for every regular uncountable $\lambda$.

It is natural to assume that the proof is more complicated than [Sh 836], but in fact it seems simpler and does not require any special background. It uses not just "abelian groups without zero" but also some derived objects, giving more leeway in the game.

Note, however, that the method here is ad-hoc, whereas [Sh 836] and HvSh 866] seem to me to be systematic. Hence their method should be helpful in more demanding related problems, in particular hopefully for fat theories (see [Sh 897]).

Received by the editors May 29, 2007, and, in revised form, September 11, 2007, and September $25,2007$.

2000 Mathematics Subject Classification. Primary 03C75.

The author's research was supported by the German-Israeli Foundation for Scientific Research and Development (Grant No. I-706-54.6/2001). Publication 907.

(C)2008 American Mathematical Society Reverts to public domain 28 years from publication 
I thank Esther Gruenhut, the referee, and John Baldwin for helpful remarks and corrections and Paul Larson for re-asking the question.

0.2. Definition. 1) We say that $M_{1}, M_{2}$ are EF-equivalent for the game of length $\alpha$ (or $\mathrm{EF}_{\alpha}$-equivalent) if $M_{1}, M_{2}$ are models (with the same vocabulary) such that the isomorphism player has a winning strategy in the game $\partial_{1}^{\alpha}\left(M_{1}, M_{2}\right)$ defined below.

1a) Replacing $\alpha$ by $<\alpha$ means: for every $\beta<\alpha$, and similarly below.

2) We say that $M_{1}, M_{2}$ are $\mathrm{EF}_{\alpha,<\mu}$-equivalent when $M_{1}, M_{2}$ are models with the same vocabulary such that the isomorphism player has a winning strategy in the game $\partial_{\mu}^{\alpha}\left(M_{1}, M_{2}\right)$ defined below.

3) For $M_{1}, M_{2}, \alpha, \mu$ as above and a partial isomorphism $f$ from $M_{1}$ into $M_{2}$ (e.g. the empty one) we define the game $\partial_{\mu}^{\alpha}\left(f, M_{1}, M_{2}\right)$ between the ISO player (the isomorphism player) and the AIS player (the anti-isomorphism player) as follows:

(a) the play lasts $\alpha$ moves;

(b) after $\beta$ moves, a partial isomorphism $f_{\beta}$ from $M_{1}$ into $M_{2}$ has been chosen increasing and continuous with $\beta$;

(c) in the $(\beta+1)$-th move, the player AIS chooses $A_{\beta, 1} \subseteq M_{1}, A_{\beta, 2} \subseteq M_{2}$ such that $\left|A_{\beta, 1}\right|+\left|A_{\beta, 2}\right|<1+\mu$, and then the player ISO chooses $f_{\beta+1} \supseteq f_{\beta}$ such that $A_{\beta, 1} \subseteq \operatorname{Dom}\left(f_{\beta+1}\right)$ and $A_{\beta, 2} \subseteq \operatorname{Rang}\left(f_{\beta+1}\right)$;

(d) if $\beta=0$, ISO chooses $f_{0}=f$; if $\beta$ is a limit ordinal, ISO chooses $f_{\beta}=$ $\bigcup\left\{f_{\gamma}: \gamma<\beta\right\}$.

The ISO player loses if he has no legal move.

4) If $f=\emptyset$, we may write $\partial_{\mu}^{\alpha}\left(M_{1}, M_{2}\right)$. If $\mu$ is 1 , we may omit it. We may write $\leq \mu$ instead of $<\mu^{+}$.

Recall:

0.3. Observation. If $\lambda=\mu^{+}$and $M, N$ are $\tau$-models and $\zeta<\lambda$ is divisible by $\lambda^{\omega}$, then

(a) if ISO wins/does not lose in $\partial_{1}^{\zeta}(M, N)$, then he wins/does not lose in $\partial_{\lambda}^{\zeta}(M, N)$;

(b) if AIS wins/does not lose in $\partial_{1}^{\zeta}(M, N)$, then he wins/does not lose in $\partial_{\lambda}^{\zeta}(M, N)$; in fact

(c) if $\mu_{1} \leq \mu_{2}$ and AIS wins/does not lose in $\partial_{\mu_{1}}^{\zeta}(M, N)$, then he wins/does not lose in $\partial_{\mu_{2}}^{\zeta}(M, N)$.

\section{Models of CARDinality $\aleph_{1}$}

1.1. Choice. 1 ) Let $G$ be a vector space of $\mathbb{Z} / 2 \mathbb{Z}$ of dimension (and cardinality) $\aleph_{0}$, with basis $\left\langle x_{n}: n<\omega\right\rangle$.

2) Let $G_{n}^{0}$ be the subspace of $G$ generated by $\left\{x_{k}: k<\omega, k \neq n\right\}$ and $G_{n}^{1}=x_{n}+G_{n}^{0}$.

3) Let $\mathcal{G}=\left\{G_{n}^{\ell}: n<\omega, \ell \in\{0,1\}\right\}$.

1.2. Observation. If $x \in G$ and $n<\omega, \ell \in\{0,1\}$, then:

(a) $x+G_{n}^{\ell}:=\left\{x+y: y \in G_{n}^{\ell}\right\} \in\left\{G_{n}^{0}, G_{n}^{1}\right\}$;

(b) $x$ has a unique representation as $x=\sum\left\{x_{k}: k \in u\right\}, u \subseteq \omega$ finite; denote $u=\operatorname{supp}(x)$

(c) $x+G_{n}^{\ell}=G_{n}^{\ell} \Leftrightarrow n \notin \operatorname{supp}(x)$. 
1.3. Construction. We define a structure $M$ :

(A) The universe of $M$ is the disjoint union of

(a) $A_{\alpha}=\{\alpha\} \times G$ for $\alpha<\omega_{1}$.

(b) $B_{\alpha}=\left\{\eta: \eta \in{ }^{\alpha} \mathcal{G}\right.$ and for some $n=n_{\eta}$ we have

$$
\aleph_{0}>\left|\left\{\beta<\alpha: \eta(\beta) \neq G_{n}^{0}\right\}\right|,
$$

where $\mathcal{G}$ is from 1.1(3) (if $\alpha \geq \omega, n_{\eta}$ is unique; if $\alpha<\omega$, let $n_{\eta}=0$ ).

So $|M|=\bigcup\left\{A_{\alpha} \cup B_{\alpha}: \alpha<\omega_{1}\right\}$, and without loss of generality the $A_{\alpha}$ 's, $B_{\alpha}$ 's are pairwise disjoint.

(B) Relations $\left(P_{1}, P_{2}\right.$ unary predicates, $F_{y}$ unary function symbol for each $y \in G$ and $E_{1}, E_{2}, R$ binary predicates):

(a) $P_{1}^{M}=\bigcup_{\alpha<\omega_{1}} A_{\alpha}$;

(b) $E_{1}^{M}=\left\{(a, b):\left(\exists \alpha \leq \beta<\omega_{1}\right)\left(a \in A_{\alpha} \wedge b \in A_{\beta}\right)\right\}$;

(c) $P_{2}^{M}=\bigcup_{\alpha} B_{\alpha}$

(d) $E_{2}^{M}=\left\{(a, b):\left(\exists \alpha \leq \beta<\omega_{1}\right)\left(a \in B_{\alpha} \wedge b \in B_{\beta}\right)\right\}$

(e) for $y \in G$ the function $F_{y}^{M}$ is defined as

( $\alpha) F_{y}^{M}((\alpha, x))=(\alpha, x+y)$ for $x \in G, \alpha<\omega_{1}$

( $\beta) F_{y}^{M} \uparrow B_{\alpha}$ is the identity (for every $\alpha<\omega_{1}$ of course);

(f) $R^{M}=\left\{(\eta,(\alpha, x))\right.$ : for some $\beta<\omega_{1}$ we have

$$
\begin{array}{ll}
(\alpha) & \eta \in B_{\beta} \subseteq{ }^{\beta} \mathcal{G}, \\
(\beta) & \alpha<\beta, \\
(\gamma) & x \in \eta(\alpha)\} .
\end{array}
$$

1.4. Definition. 1) Let $M_{<\alpha}=M \uparrow\left(\bigcup\left\{A_{\beta} \cup B_{\beta}: \beta<\alpha\right\}\right)$ for $\alpha \leq \omega_{1}$.

2) If $\gamma<\omega_{1}$ and $\nu \in{ }^{\gamma} G$ satisfy $\beta<\gamma \Rightarrow(\forall n)\left(\exists<\aleph^{\circ} \alpha<\beta\right)[n \in \operatorname{supp}(\nu(\alpha))]$, then we define $f_{\nu}$ as a function with domain $M_{<\gamma}$ by:

(a) if $(\alpha, x) \in A_{\alpha}$ and $\alpha<\gamma$, then $f_{\nu}((\alpha, x))=(\alpha, x+\nu(\alpha))$;

(b) if $\eta_{1} \in B_{\beta}$ and $\beta<\gamma$, then $f_{\nu}\left(\eta_{1}\right)=\eta_{2}$ iff

( $\alpha) \eta_{2} \in B_{\beta}$,

$(\beta)(\forall \alpha<\beta)\left(\eta_{2}(\alpha)=\nu(\alpha)+\eta_{1}(\alpha)\right)$.

Remark. 1) We can use mainly $\nu \in \gamma\left\{x_{n}: n<\omega\right\}$, a transparent case.

2) The assumption of Definition 1.4 is needed to ensure that $f_{\nu}$ maps $B_{\alpha}$ into $B_{\alpha}$.

1.5. Claim. 1) If $\gamma<\omega_{1}$ and $\nu \in{ }^{\gamma} G$ is as in Definition 1.4(2), i.e. satisfies $\beta<\gamma \Rightarrow(\forall n)\left(\exists<\aleph_{0} \alpha<\beta\right)(n \in \operatorname{supp}(\nu(\alpha))$, then

(A) $f_{\nu}$ (is well defined and) has domain $\left|M_{<\gamma}\right|$, the universe of $M_{<\gamma}$.

(B) $f_{\nu}$ is a function from $M_{<\gamma}$ into $M_{<\gamma}$.

(C) $f_{\nu}$ has range $\left|M_{<\gamma}\right|$ and is one-to-one.

(D) $f_{\nu}$ is an automorphism of $M_{<\gamma}$.

2) Conversely, if $\gamma<\omega_{1}$ and $f$ is an automorphism of $M_{<\gamma}$, then $f=f_{\nu}$ for some $\nu \in{ }^{\gamma} G$ satisfying the condition in 1.4(2).

3) If $\gamma(1)<\gamma(2)<\omega_{1}$ and $\nu_{\ell} \in{ }^{(\ell)} G$ for $\ell=1,2$ are as above and $\nu_{1} \unlhd \nu_{2}$, then $f_{\nu_{1}} \subseteq f_{\nu_{2}}$. 
Proof. 1) Clauses (A), (B):

Trivially, $f$ is a function with domain $\subseteq\left(\bigcup_{\beta<\gamma} A_{\beta}\right) \cup\left(\bigcup_{\beta<\gamma} B_{\beta}\right)$.

Clearly $\bigcup_{\beta<\gamma} A_{\beta} \subseteq \operatorname{Dom}\left(f_{\nu}\right)$ and $f_{\nu}$ maps $A_{\beta}$ into $A_{\beta} \subseteq\left|M_{<\gamma}\right|$. Let $\eta \in B_{\beta}, \beta<$ $\gamma$. Then for some $n_{*}$,

$$
u_{\eta, n_{*}}=\left\{\alpha<\beta: \eta(\alpha) \neq G_{n_{*}}^{0}\right\} \in[\beta]^{<\aleph_{0}} .
$$

Let $u_{1}=\left\{\alpha<\beta: n_{*} \in \operatorname{supp}(\nu(\alpha))\right\}$; also, this set is finite by the condition in Definition $1.4(2)$.

Let $u=u_{\eta, n_{*}} \cup u_{1}$, so $u \in[\beta]^{<\aleph_{0}}$. We define $f_{\nu}(\eta)$ as $\langle\eta(\alpha)+\nu(\alpha): \alpha<\beta\rangle$.

Now first, considering $\left\{\alpha<\beta: \eta(\alpha) \neq\left(f_{\nu}(\eta)\right)(\alpha)\right\}$ and recalling $\eta \in{ }^{\beta} \mathcal{G}$, this set is $\subseteq u$, hence is finite.

Second, if $\eta(\alpha)=G_{k}^{\ell}$, then $\left(f_{\nu}(\eta)\right)(\alpha) \in\left\{G_{k}^{0}, G_{k}^{1}\right\}$; hence $f_{\nu}(\eta) \in{ }^{\beta} \mathcal{G}$. So together $f_{\nu}(\eta) \in B_{\alpha}$.

So $B_{\beta} \subseteq \operatorname{Dom}\left(f_{\nu}\right)$ and $f_{\nu}$ maps $B_{\beta}$ into $B_{\beta}$.

\section{Clause $(\mathrm{C})$}

In fact $f_{\nu} \circ f_{\nu}=\operatorname{id}_{M_{<\gamma}}$ (the group has order 2, etc.), so should be clear. Clause (D):

Check the relations, recalling Observation 1.2

2) Let $f \in \operatorname{Aut}\left(M_{<\gamma}\right)$. The function $f$ maps $P_{1}^{M_{<\gamma}}=\bigcup_{\alpha<\gamma} A_{\alpha}$ onto itself, and by the choice of $E_{1}^{M_{<\gamma}}$ (as a quasi well ordering with the $A_{\alpha}$ as its equivalence classes) for each $\alpha<\gamma$ it maps $A_{\alpha}$ onto itself, so in particular there is $z_{\alpha}$ such that $f\left(\left(\alpha, 0_{G}\right)\right)=\left(\alpha, z_{\alpha}\right)$. Now, for every $y \in G$ by the choice of $F_{y}^{M_{<\gamma}}\left\lceil A_{\alpha}\right.$ we have $M_{<\gamma} \models F_{y}^{M_{<\gamma}}\left(\left(\alpha, 0_{G}\right)\right)=(\alpha, y)$. As $f$ is an automorphism of $M_{<\gamma}$ we also have $M_{<\gamma} \models F_{y}^{M_{<\gamma}}\left(f\left(\left(\alpha, 0_{G}\right)\right)\right)=f((\alpha, y))$ and note $F_{y}^{M_{<\gamma}}\left(\left(\alpha, z_{\alpha}\right)\right)=\left(\alpha, y+z_{\alpha}\right)$. We therefore have for every $y \in G$ that $f((\alpha, y))=F_{y}^{M_{<\gamma}}\left(\left(\alpha, z_{\alpha}\right)\right)=\left(\alpha, y+z_{\alpha}\right)$. Letting $\nu=\left\langle z_{\alpha}: \alpha<\gamma\right\rangle$ we have that $\nu \in{ }^{\gamma} G$, and it is easily verified that $f=f_{\nu}$ and that $\nu$ satisfies the condition in Definition 1.4(2).

3) Check the definition of $f_{\nu \ell}$.

1.6. Claim. Let $a_{1}=\left(0, x_{1}\right), a_{2}=\left(0, x_{0}\right) \in A_{0}$, recalling that $\left\langle x_{n}: n<\omega\right\rangle$ is a basis of $G$. If $\zeta<\omega_{1}$, then in the $E F_{\zeta}$-game for $\left(M, a_{1}\right),\left(M, a_{2}\right)$ the isomorphism player wins (this is $\partial_{1}^{\zeta}\left(\left(M, a_{1}\right),\left(M, a_{2}\right)\right)$ ).

Proof. Let $\left\langle\mathcal{U}_{\varepsilon}: \varepsilon\langle\zeta\rangle\right.$ be a partition of $\omega$ into infinite sets such that $0 \in \mathcal{U}_{0}$. In the strategy we define below, the isomorphism player does more than needed: he chooses in the $\varepsilon$-move an ordinal $\gamma(\varepsilon)>0$ and an automorphism $g_{\varepsilon}$ of $M_{<\gamma(\varepsilon)}$ mapping $a_{1}$ to $a_{2}$ and such that the elements which the anti-isomorphism player chose so far belong to $M_{<\gamma(\varepsilon)}$ (no need to distinguish domain and range). The isomorphism player ISO satisfies the following demands:

$\circledast \quad(a) \quad g_{\varepsilon}$ has form $f_{\nu_{\varepsilon}}$, where for some $\gamma(\varepsilon)<\omega_{1}$ the sequence $\nu_{\varepsilon} \in \gamma(\varepsilon)\left\{x_{n}\right.$ :

$n<\omega\}$ satisfies: $\nu_{\varepsilon}(0)=x_{0}$ and $\operatorname{Rang}\left(\nu_{\varepsilon}\right) \subseteq\left\{x_{n}: n \in \mathcal{U}_{\xi}\right.$ for some

$\xi \leq \varepsilon\}$ and $\left\langle\nu_{\varepsilon}(\alpha): \alpha<\gamma_{\varepsilon}\right\rangle$ is with no repetitions;

(b) $\langle\gamma(\xi): \xi \leq \varepsilon\rangle$ is increasing;

(c) $\left\langle\nu_{\xi}: \xi \leq \varepsilon\right\rangle$ is $\triangleleft$-increasing and $\nu(0)=x_{1}+x_{2} \in G$. 


\section{Clearly}

$(*)_{1} \quad f_{\nu_{\varepsilon}}\left(a_{1}\right)=a_{2}$ : see definition of $f_{\nu_{\varepsilon}}$ and the choice of $a_{1}, a_{2}$;

$(*)_{2} \quad f_{\nu_{\varepsilon}}$ is a partial isomorphism by $1.5(1)$;

$(*)_{3} f_{\nu_{\varepsilon}}$ extends $f_{\nu_{\xi}}$ for $\xi<\varepsilon$ by $1.5(2)$.

So ISO can satisfy the demands; hence we are done.

1.7. Claim. If $f \in \operatorname{Aut}(M)$, then $f\left\lceil A_{\alpha}=\mathrm{id}_{A_{\alpha}}\right.$ for every $\alpha<\omega_{1}$ large enough.

Proof. Let $f \in \operatorname{Aut}(M)$.

As in the proof of 1.5(2), for each $\alpha<\omega_{1}$ there is $z_{\alpha} \in G$ such that

$$
(\alpha, x) \in A_{\alpha} \Rightarrow f((\alpha, x))=\left(\alpha, z_{\alpha}+x\right) .
$$

But $G$ is countable, so for some $z_{*} \in G$ the set $\mathcal{U}:=\left\{\alpha<\omega_{1}: z_{\alpha}=z_{*}\right\}$ is unbounded in $\omega_{1}$, and if possible choose $z_{*}$ such that it is $\neq 0_{G}$; let $u$ be such that $z_{*}=\sum\left\{x_{n}: n \in u\right\}$, so $u \subseteq \omega$ is finite.

Hence we can find $\gamma_{*}<\omega_{1}$ such that $\mathcal{U} \cap \gamma_{*}$ is infinite. If $z_{*} \neq 0_{G}$, let $n \in u=$ $\operatorname{supp}\left(z_{*}\right)$ and let $\eta \in B_{\gamma_{*}}$ be constantly $G_{n}^{0}$; such an $\eta$ exists by the definition of $B_{\gamma_{*}}$. Now $f(\eta)$ is "illegal", i.e. satisfies $\left\{\alpha<\gamma_{*}: f(\eta)(n) \neq G_{n}^{0}\right\}$ is infinite, contradicting Construction 1.3(A)(b). So $z_{*}=0_{G}$; hence by the choice of $z_{*}$ we have $z_{\gamma}=0_{G}$ for every $\gamma<\omega_{1}$ large enough, say for $\gamma \in\left[\gamma_{*}, \omega_{1}\right)$, i.e. $f\left\lceil A_{\gamma}=\mathrm{id}_{A_{\gamma}}\right.$, so we are done.

1.8. Definition. 1) For a sequence $\mathbf{p}=\left\langle\left(\beta_{\alpha}, g_{\alpha}\right): \alpha<\omega_{1}\right\rangle$ satisfying $\beta_{\alpha} \leq \alpha, g_{\alpha} \in$ $\operatorname{Hom}(G, G)$ we define $M_{\mathbf{p}}$ as the expansion of $M$ by $R_{1}^{M_{\mathbf{p}}}=\left\{\left(\left(\beta_{\alpha}, y_{2}\right),\left(\alpha, y_{1}\right)\right)\right.$ : $\left.\alpha<\omega_{1}, y_{2}, y_{1} \in G, g_{\alpha}\left(y_{1}\right)=y_{2}\right\}$.

1a) Let $\mathbf{P}$ be the set of such $\mathbf{p}$ 's.

2) For a sequence $\mathbf{p}=\left\langle\left(\beta_{\alpha}, h_{\alpha}\right): \alpha<\omega_{1}\right\rangle$ satisfying $\beta_{\alpha} \leq \alpha$ and $h_{\alpha} \in{ }^{\omega} \omega$, the model $M_{\mathbf{p}}$ is defined as $M_{\left\langle\left(\beta_{\alpha}, \hat{h}_{\alpha}\right): \alpha<\omega_{1}\right\rangle}$, where $\hat{h}_{\alpha}$ is the homomorphism from $G$ to $G$ defined by $\hat{h}_{\alpha}\left(\sum_{n \in u} x_{n}\right)=\sum_{n \in u} x_{h_{\alpha}(n)}$. Let $\mathbf{P}^{\prime}$ be the set of such $\mathbf{p}$ 's.

3) Let $M_{<\gamma}^{\mathbf{p}}=M_{\mathbf{p}}\left\lceil\left(\left|M_{<\gamma}\right|\right)\right.$ for $\gamma<\omega_{1}$ and $\mathbf{p}$ as above.

1.9. Claim. If $\mathbf{p}=\left\langle\left(\beta_{\alpha}, g_{\alpha}\right): \alpha \in \omega_{1}\right\rangle \in \mathbf{P}$ is as in Definition 1.8, $\gamma<\omega_{1}$ and $\nu \in{ }^{\gamma} G$ is as in Definition 1.4 and $f_{\nu}$ is as in Definition 1.4, then $f_{\nu}$ is an automorphism of $M_{<\gamma}^{\mathbf{p}}$ iff

$\circledast \mathbf{p}, \nu \quad$ if $\alpha<\gamma$, then $g_{\alpha}$ maps $\nu(\alpha)$ to $\nu\left(\beta_{\alpha}\right)$.

Proof. Straightforward. For $\alpha<\gamma$ and $y_{1}, y_{2} \in G$ we have:

(a) $\left(f_{\nu}\left(\left(\beta_{\alpha}, y_{2}\right)\right), f_{\nu}\left(\left(\alpha, y_{1}\right)\right)\right) \in R_{1}^{M_{\mathbf{p}}}$ iff (by $f_{\nu}$ 's definition),

(b) $\left(\left(\beta_{\alpha}, y_{2}+\nu\left(\beta_{\alpha}\right)\right),\left(\alpha, y_{1}+\nu(\alpha)\right)\right) \in R_{1}^{M_{\mathbf{p}}}$ iff (by $R_{1}^{M_{\mathrm{p}}}$ 's definition),

(c) $g_{\alpha}\left(y_{1}+\nu(\alpha)\right)=\left(y_{2}+\nu\left(\beta_{\alpha}\right)\right)$

iff (as $g_{\alpha}$ is an endomorphism of $G$ ),

(d) $g_{\alpha}\left(y_{1}\right)+g_{\alpha}(\nu(\alpha))=y_{2}+\nu\left(\beta_{\alpha}\right)$.

So if $\circledast_{\mathbf{p}, \nu}$ holds, then clause (d) is equivalent to:

(e) $g_{\alpha}\left(y_{1}\right)+\nu\left(\beta_{\alpha}\right)=y_{2}+\nu\left(\beta_{\alpha}\right)$

iff (by cancellation), 
(f) $g_{\alpha}\left(y_{1}\right)=y_{2}$

iff (by the definition of $R_{1}^{M_{\mathbf{p}}}$ ),

(g) $\left(\left(\beta_{\alpha}, y_{2}\right),\left(\alpha, y_{1}\right)\right) \in R_{1}^{M_{\mathbf{p}}}$.

So $\circledast_{\mathbf{p}, \nu}$ implies $f_{\nu}$ is an automorphism of $M_{<\gamma}^{\mathbf{p}}$. The inverse is easy, too. $\quad \square_{1.9}$

1.10. Claim. Let

(a) $h_{\alpha}: \omega \rightarrow \omega$ be $h_{\alpha}(2 n+\ell)=\ell$ for $n<\omega, \ell \in\{0,1\}$ and $\alpha<\omega_{1}$;

(b) $\mathbf{p}=\left\langle\left(0, h_{\alpha}\right): \alpha<\omega_{1}\right\rangle \in \mathbf{P}^{\prime}$, so $\beta_{\alpha}=0$ for every $\alpha$;

(c) $N=M_{\mathbf{p}}$;

(d) $\Lambda_{\gamma}=\left\{\nu \in{ }^{\gamma} G: \nu(0)=x_{0}+x_{1}\right.$ and if $\beta \in(0, \gamma)$, then $\nu(\beta)$ has the form $x_{2 n}+x_{2 n+1}$ and $\langle\nu(\beta): \beta<\gamma\rangle$ is with no repetitions $\}$.

$\underline{\text { Then }}$

(A) If $\nu \in \Lambda_{\gamma}, \gamma<\omega_{1}$, then $f_{\nu}$ is an automorphism of $M_{<\gamma}^{\mathbf{p}}$ extending $f_{\nu \uparrow \beta}$ for $\beta<\gamma$.

$(A)^{\prime}$ For $\zeta<\omega_{1}$, in the game $\partial^{\zeta}\left(\left(N,\left(0, x_{0}\right)\right),\left(N,\left(0, x_{1}\right)\right)\right.$, the isomorphism player wins; i.e. $\left(N,\left(0, x_{1}\right)\right),\left(N,\left(0, x_{2}\right)\right)$ are $E F_{\zeta}$-equivalent.

(B) $N$ has no automorphism mapping $a_{1}=\left(0, x_{0}\right)$ to $a_{2}=\left(0, x_{1}\right)$.

$(B)^{\prime}\|N\|=\aleph_{1}$, so the isomorphism player loses $\partial^{\omega_{1}}\left(\left(M, a_{1}\right),\left(M, a_{2}\right)\right)$.

Proof.

Clauses (A), (A)': As in Claim 1.6, using Claim 1.9.

Clauses (B). (B)': Toward a contradiction assume $f \in \operatorname{Aut}\left(M_{\mathbf{p}}\right), f\left(\left(0, x_{0}\right)\right)=$ $\left(0, x_{1}\right)$. Continue as in the proof of Claim 1.7, but $z_{\alpha} \neq 0$ for $\alpha<\omega_{1}$ (as $\left.\hat{h}_{\alpha}\left(z_{\alpha}\right)=x_{0}+x_{1}\right)$; hence $z_{*}$ is not zero and we get a contradiction. $\quad \square_{1.10}$

1.11. Remark. We can in Claim 1.10 get a rigid $M$ : of interest?

1.12. Conclusion. There are non-isomorphic models of cardinality $\aleph_{1}$ which are $\mathrm{EF}_{\zeta}$-equivalent for every $\zeta<\omega_{1}$.

\section{OTHER CARDINALS}

2.1. Claim. 1) If $\lambda=\mu^{+}$, then there are models $M, N$ of cardinality $\lambda, E F_{\zeta^{-}}$ equivalent for every $\zeta<\lambda$ but not isomorphic, hence not $E F_{\lambda}$-equivalent.

2) Instead of $\lambda=\mu^{+}$, just $\lambda=\operatorname{cf}(\lambda)=\mu>\aleph_{0}$ is enough.

Remark. 1) For $\lambda$ regular uncountable not a successor, it makes a difference whether we allow the anti-isomorphism player to choose one element or $<\lambda$. In Claim 2.1(2) we allow one element.

2) For Claim 2.1 recall Observation 0.3.

Proof. 1) Now let $G=\bigoplus\left\{(\mathbb{Z} / 2 \mathbb{Z}) x_{\varepsilon}: \varepsilon<\mu\right\}$ and repeat 1.1-1.10 with the obvious changes: $\aleph_{0}, \aleph_{1}$ replaced by $\mu, \lambda$ but "finite" remains "finite", in particular in Construction $1.3(\mathrm{~A})(\mathrm{b})$.

2) So without loss of generality $\lambda$ is not a successor cardinal (hence is (possibly weakly) inaccessible). Define the abelian group $G$ as in part (1), but now $\mu=\lambda$ and repeat 1.1-1.5, and also 1.8, 1.9 as above, but now $h \in{ }^{\lambda} \lambda$. But to imitate Claim 1.10 we choose $\mathbf{p}$ differently. Let $\mathcal{U}_{\varepsilon}=\left[\gamma_{\varepsilon}, \gamma_{\varepsilon+1}\right)$ for $\varepsilon<\lambda$, where $\left\langle\gamma_{\varepsilon}: \varepsilon\langle\lambda\rangle\right.$ is increasing continuous, $\gamma_{0}=0, \gamma_{1}=2$, each $\gamma_{\varepsilon}$ is even and $\gamma_{\varepsilon+1}=\gamma_{\varepsilon}+2 \varepsilon$. So let $\left\langle\mathcal{U}_{\varepsilon}: \varepsilon<\lambda\right\rangle$ 
be a partition of $\lambda$ into sets such that $\left|\mathcal{U}_{\varepsilon}\right|=|2 \varepsilon|$. Let $\mathbf{p}=\left\langle\beta_{\alpha}, h_{\alpha}: \alpha<\lambda\right\rangle$ be chosen as follows $\left(\beta_{\alpha} \leq \alpha\right.$ and $h_{\alpha} \in{ }^{\lambda} \lambda$ of course and):

(a) let $h_{k}(2 \alpha+\ell)=\ell$ for $\alpha<\lambda, k<2, \ell<2$, and let $\beta_{0}=\beta_{1}=0$;

(b) for $\alpha \in[2, \lambda)$ let $\beta_{\alpha}=1$ and $h_{\alpha}$ be such that for $\ell \in\{0,1\}$ and $\gamma<\lambda$ we have $2 \gamma+\ell \in \mathcal{U}_{\varepsilon} \Rightarrow h_{\alpha}(2 \gamma+\ell)=2 \varepsilon+\ell$.

To prove the parallel of clause (B) of Claim 1.10 toward a contradiction, assume that $\left(M_{\mathbf{p}}, a_{1}\right),\left(M_{\mathbf{p}}, a_{2}\right)$ are isomorphic. Let $f$ be such an isomorphism, so $f$ is an automorphism of $M_{\mathbf{p}}$ mapping $a_{1}$ to $a_{2}$. As in the proof of Claim 1.7, we can find $z_{\alpha} \in G$ for $\alpha<\lambda$ such that $x \in G \Rightarrow f((\alpha, x))=\left(\alpha, z_{\alpha}+x\right)$. Let $\nu=\left\langle z_{\alpha}: \alpha<\lambda\right\rangle \in{ }^{\lambda} G$. So by the parallel to Claim 1.9 we know that $\circledast_{\mathbf{p}, \nu \mid \gamma}$ holds for $\gamma<\lambda$, i.e. $\hat{h}_{\alpha}(\nu(\alpha))=\nu\left(\beta_{\alpha}\right)$.

But $f\left(a_{1}\right)=a_{2}$, so $z_{0}=x_{0}+x_{1}$; hence recalling $\beta_{1}=0$ we have $\nu(1)=z_{1} \neq 0$, and let $\varepsilon<\lambda, \ell<2$ be such that $2 \varepsilon+1 \in \operatorname{supp}\left(z_{1}\right)$. By the end of the previous paragraph,

$(*)$ if $2 \leq \alpha<\lambda$, then $\operatorname{supp}\left(z_{\alpha}\right) \cap\left[\gamma_{\varepsilon}, \gamma_{\varepsilon+1}\right) \neq \emptyset$; hence we can find $\gamma^{\alpha} \in$ $\operatorname{supp}\left(z_{\alpha}\right) \cap\left[\gamma_{\varepsilon}, \gamma_{\varepsilon+1}\right)$.

So for some $\gamma_{*}<\lambda$ the set $\mathcal{U}=\left\{\alpha<\lambda: \gamma_{*}=\gamma^{\alpha}\right.$; hence $\left.\gamma_{*} \in \operatorname{supp}\left(z_{\alpha}\right)\right\}$ is an unbounded subset of $\lambda$. We continue as in the proof of Claim 1.7.

Let $\varepsilon<\lambda$. Without loss of generality, $\varepsilon \geq \omega$ is a cardinal, and we shall prove that $\left(M_{\mathbf{p}}, a_{1}\right),\left(M_{\mathbf{p}}, a_{2}\right)$ are $\mathrm{EF}_{\varepsilon}$-equivalent. We act as in the proof of Claim 1.6 (though $a_{1}, a_{2}$ are different), but instead of $M_{<\gamma}$ there we use here $M_{u}^{\mathbf{p}}$, where $M_{u}^{\mathbf{p}}=M_{\mathbf{p}}\left\lceil\bigcup\left\{A_{\alpha} \cup B_{\alpha}: \alpha \in u\right\}\right.$ for $u \subseteq \lambda$.

Let $u_{0}=\{0,1\}$. In stage $\zeta<\varepsilon$ of the game the AIS player chooses $\ell(\zeta) \in\{1,2\}$ and $b_{\zeta}^{\ell(\zeta)} \in\left(M_{\mathbf{p}}, a_{\ell(\zeta)}\right)$. So there is $\xi_{\zeta}<\lambda$ such that $b_{\zeta}^{\ell(\zeta)} \in A_{\xi_{\zeta}} \cup B_{\xi_{\zeta}}$. The ISO player chooses $u_{\zeta+1} \in[\lambda]^{<\varepsilon}$ and a sequence $\bar{\beta}_{\zeta+1}=\left\langle\beta_{\alpha}: \alpha \in u_{\zeta+1} \backslash\{0,1\} \wedge 2 \beta_{\alpha} \in\right.$ $\left.\mathcal{U}_{\varepsilon}\right\rangle$ satisfying the following:

(a) $u_{\zeta+1}=u_{\zeta} \cup\left\{\xi_{\zeta}\right\}$,

(b) $\bar{\beta}_{\zeta}=\bar{\beta}_{\zeta+1}\left\lceil u_{\zeta} \backslash\{0,1\}\right.$,

(c) $\bar{\beta}_{\zeta+1}$ is with no repetitions.

For $\nu_{\zeta+1} \in{ }^{u_{\zeta+1}} G$ defined by

$$
\nu_{\zeta+1}(\alpha)= \begin{cases}x_{0}+x_{1} & \alpha=0 \\ x_{2 \varepsilon}+x_{2 \varepsilon+1} & \alpha=1 \\ x_{2 \beta_{\alpha}}+x_{2 \beta_{\alpha}+1} & \text { else }\end{cases}
$$

$f_{\nu_{\zeta+1}}$ satisfies the requirement in Definition 1.4(2) (with the modification $\forall \beta \in u_{\zeta+1}$ instead of $\forall \beta<\gamma$ ) and hence is an automorphism of $M_{u_{\zeta+1}}^{\mathbf{p}}$ and $f_{\nu_{\zeta+1}}\left(a_{1}\right)=a_{2}$.

\section{REFERENCES}

[HvSh 866] Chanoch Havlin and Saharon Shelah. Existence of EF-equivalent non-isomorphic models. Mathematical Logic Quarterly, 53:111-127, 2007. MR2308491 (2008d:03029)

[Sh 897] Saharon Shelah. Theories with EF-Equivalent Non-isomorphic Models. Tbilisi Mathematical Journal, submitted. math.LO/0703477.

[Sh 836] Saharon Shelah. On long EF-equivalence in non-isomorphic models. In Proceedings of Logic Colloquium, Helsinki, August 2003, Lecture Notes in Logic, volume 24, pages 315-325. Assoc. Symbol. Logic, 2006. MR2207360 (2007a:03044) 
[Tur90] Heikki Tuuri. Infinitary languages and Ehrenfeucht-Fraïssé games. Ph.D. thesis, University of Helsinki, 1990.

[Va95] Jouko Väänänen. Games and trees in infinitary logic: A survey. In M. Mostowski M. Krynicki and L. Szczerba, editors, Quantifiers, pages 105-138. Kluwer, 1995.

Einstein Institute of Mathematics, The Hebrew University of Jerusalem, Edmond J. Safra Campus, Givat Ram, Jerusalem 91904, Israel - And - Department of Mathematics, Hill Center, Rutgers, The State University of New Jersey, 110 Frelinghuysen Road, Piscataway, New Jersey 08854-8019

E-mail address: shelah@math.huji.ac.il 